Edinburgh 2006/15

August 2006

\title{
Multi-Higgs boson production in the Standard Model and beyond
}

\author{
T. Binoth ${ }^{a}$, S. $\operatorname{Karg}^{b}$, N. Kauer ${ }^{b}$ and R. Rückl ${ }^{b}$ \\ ${ }^{a}$ School of Physics, The University of Edinburgh, \\ Edinburgh EH9 3JZ, Scotland UK \\ ${ }^{b}$ Institut für Theoretische Physik und Astrophysik, \\ Universität Würzburg, D-97074 Würzburg, Germany
}

\begin{abstract}
We present a calculation of the loop-induced processes $g g \rightarrow H H$ and $g g \rightarrow H H H$, and investigate the observability of multi-Higgs boson production at the CERN Large Hadron Collider (LHC) in the Standard Model (SM) and beyond. While the SM cross sections are too small to allow observation at the LHC, we demonstrate that physics beyond the SM can lead to amplified, observable cross sections. Furthermore, the applicability of the heavy top quark approximation in two- and three-Higgs boson production is investigated. We conclude that multi-Higgs boson production at the SuperLHC is an interesting probe of Higgs sectors beyond the SM and warrants further study.
\end{abstract}




\section{Introduction}

The understanding of electroweak symmetry breaking is a major goal of the physics programme at the upcoming Large Hadron Collider (LHC) at CERN. In the Standard Model (SM), electroweak symmetry breaking is mediated by the Higgs mechanism, which predicts a fundamental scalar particle, the Higgs boson [1]. At the LHC, the discovery of the Higgs boson is feasible in the entire theory-compatible mass region $[2,3]$. Theoretically, a heavy Higgs boson with mass of order $1 \mathrm{TeV}$ in the vicinity of the non-perturbative regime is still feasible $[4,5]$. Electroweak precision measurements, however, favour a relatively light SM Higgs boson not too far above the direct LEP exclusion limit of $114.4 \mathrm{GeV}$ [6].

In order to establish the Higgs mechanism and confirm the Higgs sector of the SM, the discovery of a Higgs-like boson is not sufficient. In addition, the predicted couplings of fermions and gauge bosons to the Higgs boson, as well as the trilinear and quartic Higgs self couplings need to be confirmed experimentally. While Higgs-fermion and Higgs-gauge boson couplings are measurable with accuracies of $10-40 \%$ at the LHC [7] and in many channels considerably better at the International Linear Collider (ILC) [8], the measurement of the trilinear and quartic Higgs self couplings, which are probed in double and triple Higgs boson production, respectively, are more challenging. For low Higgs masses not too much above the LEP limit $\left(m_{H} \lesssim 140 \mathrm{GeV}\right)$, the largest rates are obtained with the decay channel $H \rightarrow b \bar{b}$ dominating in this mass regime. Unfortunately, at the LHC this search channel is not viable, because of an overwhelming QCD background. The ILC, however, would allow the measurement of the trilinear Higgs self coupling to a precision of 20-30\% in the low Higgs mass regime [8-11]. We note that in this regime a photon collider promises an even better determination of the trilinear Higgs coupling [12-14]. For higher Higgs masses, the ILC production cross section decreases due to the reduced phase space. However, in this mass region, the vector boson pair decay channels open up and allow for leptonic signatures that can be separated from the backgrounds at the LHC $[8,15-19]$.

The dominant production mechanism for Higgs boson pairs at the LHC is gluon fusion. We note that Higgs production in hadronic collisions can also proceed through bottom quark fusion, $b \bar{b} \rightarrow n H$, but in the SM the corresponding LO as well as NLO [20] cross sections are negligible. In the MSSM, however, enhanced Yukawa couplings can lead to comparable cross sections for gluon and bottom quark fusion [21].

The gluon fusion loop amplitude was first presented in [22]. For neutral Higgs boson pairs in the Minimal Supersymmetric Standard Model (MSSM) the top/bottom loop contribution was evaluated in $[23,24]$. Charged Higgs boson pairs including squark effects were studied in [25]. Although the SM cross section for triple Higgs boson production at the LHC can be expected to be small $[9,10]$, this expectation has to be verified through explicit calculation. Only recently, a full calculation of the process $g g \rightarrow H H H$ appeared in the literature [26] and confirmed that SM cross sections are indeed too small to be observable at the LHC. However, it has to be stressed that multi-Higgs boson production rates are very sensitive to physics beyond the Standard Model (BSM) and should be scrutinised carefully in high energy experiments, as they might point to new physics at high energy scales that are not directly accessible at the given collider [27,28]. A non-standard heavy quark that receives its mass via the Higgs mechanism does not decouple [29], and therefore leads to a non-vanishing contribution in heavy quark loop-induced processes. Furthermore, contributions of higher dimensional operators might alter the SM cross section considerably [30]. Also, in certain Little Higgs Models the Higgs pair production cross section is significantly different from the SM cross section [31].

In this paper, we present our calculation of double and triple Higgs boson production via gluon fusion. This provides an independent check of the recent calculation in [26], which employed different computational methods and tools. To go beyond the findings of [26], we study higher dimensional operator effects on production rates relative to the SM. The corresponding couplings are in principle only restricted by unitarity constraints [32]. We also analyse amplification effects in supersymmetric 
(SUSY) two-Higgs-doublet models (2HDMs).

The paper is organised as follows. After briefly reviewing Higgs boson properties relevant for our investigation in Section 2, we describe our calculation in Section 3. In Section 4, SM and BSM results are presented and discussed. Section 5 gives a brief summary.

\section{Higgs boson properties}

The SM Higgs mechanism provides fundamental mass terms for massive vector bosons and fermions. The coupling strength of the Higgs boson is proportional to the mass (squared) of the interacting fermion (gauge boson). The SM Higgs boson self interactions, induced by the scalar potential

$$
V=\frac{m_{H}^{2}}{2 v^{2}}\left(\Phi^{\dagger} \Phi-\frac{v^{2}}{2}\right)^{2}
$$

are also proportional to the Higgs mass squared. In unitary gauge one has

$$
V=\frac{m_{H}^{2}}{2} H^{2}+\frac{\lambda_{3}}{3 !} H^{3}+\frac{\lambda_{4}}{4 !} H^{4}
$$

with

$$
\lambda_{4}=\lambda_{3} / v=\frac{3 m_{H}^{2}}{v^{2}} .
$$

Relation (3) between the trilinear and quartic Higgs self couplings is a genuine SM prediction. To establish the SM Higgs mechanism, it has to be verified experimentally. As already pointed out, the discovery of the Higgs boson and the measurement of its couplings to fermions and gauge bosons alone are not sufficient.

In general, the Higgs self couplings change in extensions of the SM. By allowing for higher dimensional operators of the type

$$
\sum_{k=1}^{\infty} \frac{g_{k}}{\Lambda^{2 k}}\left(\Phi^{\dagger} \Phi-\frac{v^{2}}{2}\right)^{2+k}
$$

the constraint (3) is relaxed. Magnitude and sign of $\lambda_{3}$ and $\lambda_{4}$ can be arbitrary up to constraints imposed by unitarity. In order to guarantee the stability of the vacuum, only the sign of the highest power of the Higgs field has to be positive. We note that the addition of singlet Higgs fields preserves relation (3), but may lead to invisible Higgs boson decays and diluted Higgs signals [33].

The ability to measure the Higgs self couplings depends on the size of multi-Higgs boson cross sections. As will be discussed below, SM rates are very small at the LHC (see also [26]). It is thus interesting to consider extensions of the SM that allow for amplified event rates. For Higgs pair production in gluon fusion this has been studied in [23] in the context of the MSSM. Two amplification sources have been identified. Firstly, the top and bottom Yukawa couplings are altered due to the mixing of the Higgs fields. In the MSSM one has, at tree level,

$$
\begin{aligned}
& \lambda_{h t \bar{t}}=\frac{m_{t}}{v} \frac{\cos \alpha}{\sin \beta}, \\
& \lambda_{h b \bar{b}}=-\frac{m_{b}}{v} \frac{\sin \alpha}{\cos \beta},
\end{aligned}
$$

where

$$
\tan \beta=\frac{v_{2}}{v_{1}} \quad \text { and } \quad \alpha=\frac{1}{2} \arctan \left(\frac{M_{A}^{2}+M_{Z}^{2}}{M_{A}^{2}-M_{Z}^{2}} \tan 2 \beta\right), \quad-\frac{\pi}{2} \leq \alpha \leq 0 .
$$


Here, $v_{1}\left(v_{2}\right)$ is the vacuum expectation value of the Higgs doublet with weak hypercharge $-\frac{1}{2}\left(+\frac{1}{2}\right)$. For sufficiently large $\tan \beta$, the bottom-loop contribution to the cross section becomes sizable in comparison to the top-loop contribution, leading to a larger production rate. Secondly, internal Higgs propagators can become resonant thereby enhancing the production rate. Both effects generally play a role in $2 \mathrm{HDMs}$, as will be discussed and quantified for triple Higgs boson production below.

We will now briefly review the features of $2 \mathrm{HDM}$ s that are important for our purposes. The general potential of the $2 \mathrm{HDM}$ is given by $[34,35]$

$$
\begin{aligned}
V\left(\Phi_{1}, \Phi_{2}\right)= & m_{11}^{2} \Phi_{1}^{\dagger} \Phi_{1}+m_{22}^{2} \Phi_{2}^{\dagger} \Phi_{2}-\left(m_{12}^{2} \Phi_{1}^{\dagger} \Phi_{2}+\text { h.c. }\right)+ \\
& +\frac{\lambda_{1}}{2}\left(\Phi_{1}^{\dagger} \Phi_{1}\right)^{2}+\frac{\lambda_{2}}{2}\left(\Phi_{2}^{\dagger} \Phi_{2}\right)^{2}+ \\
& +\lambda_{3}\left(\Phi_{1}^{\dagger} \Phi_{1}\right)\left(\Phi_{2}^{\dagger} \Phi_{2}\right)+\lambda_{4}\left(\Phi_{1}^{\dagger} \Phi_{2}\right)\left(\Phi_{2}^{\dagger} \Phi_{1}\right)+ \\
& +\left\{\frac{\lambda_{5}}{2}\left(\Phi_{1}^{\dagger} \Phi_{2}\right)^{2}+\left[\lambda_{6}\left(\Phi_{1}^{\dagger} \Phi_{1}\right)+\lambda_{7}\left(\Phi_{2}^{\dagger} \Phi_{2}\right)\right]\left(\Phi_{1}^{\dagger} \Phi_{2}\right)+\text { h.c. }\right\}
\end{aligned}
$$

with the complex Higgs-doublet fields acquiring the vacuum expectation values $v_{1}$ and $v_{2}$. After diagonalising the mass matrix one obtains the physical Higgs fields $h, H, A, H^{ \pm}$and the Goldstone bosons $G^{ \pm}, G$. We are interested in the Higgs self couplings, which can be written in terms of the dimensionless parameters $\lambda_{i}, i=1, \ldots, 7$, appearing in (7). For the quartic couplings, one has

$$
\begin{aligned}
\lambda_{h h h h}= & 3 \cos ^{4} \alpha \lambda_{2}+3 \sin ^{4} \alpha \lambda_{1}+6 \cos ^{2} \alpha \sin ^{2} \alpha\left(\lambda_{3}+\lambda_{4}+\lambda_{5}\right) \\
& -12 \cos ^{3} \alpha \sin \alpha \lambda_{7}-12 \sin ^{3} \alpha \cos \alpha \lambda_{6}, \\
\lambda_{H h h h}= & -3 \cos \alpha \sin ^{3} \alpha \lambda_{1}+3 \cos ^{3} \alpha \sin \alpha \lambda_{2}-\frac{3}{2} \cos 2 \alpha \sin 2 \alpha\left(\lambda_{3}+\lambda_{4}+\lambda_{5}\right) \\
& +3\left(3 \cos ^{2} \alpha \sin ^{2} \alpha-\sin ^{4} \alpha\right) \lambda_{6}+3\left(\cos ^{4} \alpha-3 \cos ^{2} \alpha \sin ^{2} \alpha\right) \lambda_{7},
\end{aligned}
$$

to be multiplied by $M_{Z}^{2} / v^{2}$. In the MSSM, the Higgs sector is constrained such that only two of the seven input parameters are free. Choosing $\tan \beta$ and $M_{A}=\lambda_{6} v^{2}$ as basic input, one recovers the MSSM values

$$
\begin{aligned}
\lambda_{h h h h} & =3 \cos ^{2} 2 \alpha, \\
\lambda_{H h h h} & =3 \cos 2 \alpha \sin 2 \alpha .
\end{aligned}
$$

As is well known, radiative corrections in the Higgs sector are large. The largest ubiquitous correction is given by $3 G_{F} m_{t}^{4} /\left(\sqrt{2} \pi^{2} \sin ^{2} \beta\right) \cdot \ln \left(m_{\tilde{t}}^{2} / m_{t}^{2}\right)$. In our numerical analysis for the MSSM all one-loop and leading two-loop corrections to Higgs masses and couplings are included [36,37]. For a discussion about reconstructing the Higgs potential in the SUSY case, see [38].

\section{Calculation}

From a computational point of view loop amplitudes with five or more external legs are challenging due to their combinatorial complexity. We only sketch here our method, which leads to a numerically stable algebraic representation of the amplitude. More details can be found in [39-44].

\subsection{Structure of the amplitude}

The production processes $p p \rightarrow h h$ and $p p \rightarrow h h h$ proceed at the parton level via gluon fusion in combination with a quark loop. Here $h$ stands for the Higgs boson of the SM or the light CP-even Higgs boson of the MSSM. Squarks in the loop have been neglected. Their contributions vanish for 
large squark masses, due to their decoupling property. In the SM, only the top quark loop leads to a non-negligible cross section at the LHC. For triple Higgs boson production,

$$
g\left(p_{1}, \lambda_{1}\right)+g\left(p_{2}, \lambda_{2}\right) \rightarrow h\left(p_{3}\right)+h\left(p_{4}\right)+h\left(p_{5}\right),
$$

with $p_{i}$ defining the 4-momenta and $\lambda_{1,2}$ specifying the gluon helicities, the Feynman diagrams are classified in Fig. 1. On the diagrammatic level 3-, 4- and 5-point topologies can be distinguished. Each topology involves a different combination of coupling constants. Here, we only discuss the

$P$ :

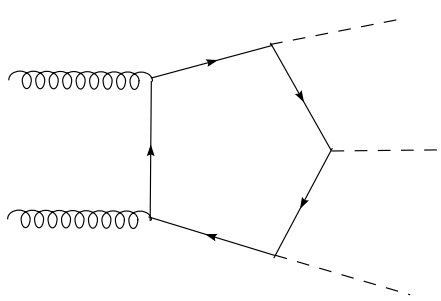

$T_{1}$ :

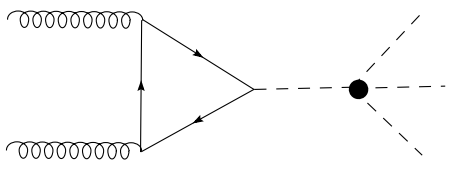

$B:$

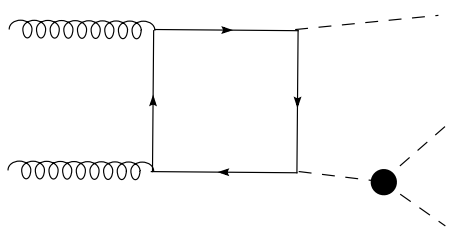

$T_{2}$ :

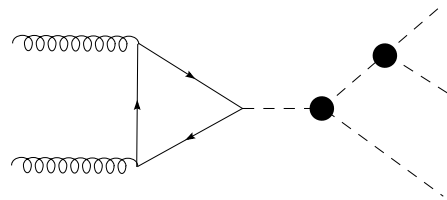

Figure 1: Illustration of different topologies to the process $g g \rightarrow h h h$.

structure of the SM amplitude. In 2HDMs one has additional tree structures from the heavy Higgs boson $H$ attached to the quark loop. The pentagon topology $P$ contains no Higgs self coupling. The box topologies $B$ are proportional to $\lambda_{3}$, whereas the triangle topologies $T_{1}$ and $T_{2}$ are proportional to $\lambda_{4}$ and $\lambda_{3}^{2}$, respectively. Subsequently, the amplitude can be expressed as

$$
\begin{gathered}
\Gamma(g g \rightarrow h h h)=\delta_{a b} T_{R} \frac{\alpha_{s}}{4 \pi} \varepsilon_{1, \mu} \varepsilon_{2, \nu} \mathcal{M}^{\mu \nu} \\
\mathcal{M}^{\mu \nu}=\lambda_{t t h}^{3} \mathcal{M}_{P}^{\mu \nu}+\lambda_{3} \lambda_{t t h}^{2} \mathcal{M}_{B}^{\mu \nu}+\lambda_{4} \lambda_{t t h} \mathcal{M}_{T, 1}^{\mu \nu}+\lambda_{3}^{2} \lambda_{t t h} \mathcal{M}_{T, 2}^{\mu \nu} .
\end{gathered}
$$

The scattering tensor $\mathcal{M}^{\mu \nu}$ can be decomposed in terms of metric tensors and external momenta and, using momentum conservation, be expressed as

$$
\mathcal{M}^{\mu \nu}=\hat{A} g^{\mu \nu}+\sum_{j, l=1,4} \hat{B}_{j l} p_{j}^{\mu} p_{l}^{\nu} .
$$

By solving the Ward identities $\mathcal{M}^{\varepsilon_{1} p_{2}}=0, \mathcal{M}^{p_{1} \varepsilon_{2}}=0$, or equivalently using axial gauge conditions (e.g. $\varepsilon_{1} \cdot p_{2}, \varepsilon_{2} \cdot p_{1}$ ), one can achieve a manifestly gauge invariant representation of the amplitude. Introducing the Abelian part of the gluon field strength tensor $\mathcal{F}_{j}^{\mu \nu}=\varepsilon_{j}^{\mu} p_{j}^{\nu}-p_{j}^{\mu} \varepsilon_{j}^{\nu}$ it reads

$$
\mathcal{M}^{\varepsilon_{1} \varepsilon_{2}}=A \operatorname{tr}\left(\mathcal{F}_{1} \mathcal{F}_{2}\right)+\sum_{j, l=3,4} B_{j l} p_{2} \cdot \mathcal{F}_{1} \cdot p_{j} p_{1} \cdot \mathcal{F}_{2} \cdot p_{l}
$$


The amplitude coefficients $A, B_{j l}$ are equal to $\hat{A}, \hat{B}_{j l}$, up to trivial factors. Bose symmetry of the gluons and Higgs bosons leads to additional relations between them. After determining all amplitude coefficients, verifying the Ward identities and Bose symmetry served as a powerful check of our calculation.

It is useful to decompose the amplitude further into helicity components. Due to parity invariance only two helicity amplitudes have to be known:

$$
\begin{aligned}
& \mathcal{M}^{--}=\mathcal{M}^{++} \\
& \mathcal{M}^{-+}=\mathcal{M}^{+-}
\end{aligned}
$$

Applying spinor helicity methods $[45,46]$, the polarisation vectors for \pm helicities are given by

$$
\begin{aligned}
\varepsilon_{1}^{+\mu} \varepsilon_{2}^{+\nu} & =-\frac{[21]}{\langle 12\rangle} \frac{\operatorname{tr}^{-}(1 \nu 2 \mu)}{2 s_{12}} \\
\varepsilon_{1}^{+\mu} \varepsilon_{2}^{-\nu} & =\frac{\left\langle 2^{-}|\mu| 1^{-}\right\rangle}{\sqrt{2}\langle 21\rangle} \frac{\left\langle 2^{-}|\nu| 1^{-}\right\rangle}{\sqrt{2}[12]}
\end{aligned}
$$

with $\operatorname{tr}^{-}(1 \nu \ldots) \equiv\left[\operatorname{tr}\left(\not p_{1} \gamma^{\nu} \ldots\right)-\operatorname{tr}\left(\gamma_{5} \not \not \gamma_{1} \gamma^{\nu} \ldots\right)\right] / 2$ and the spinor inner products $\langle i j\rangle \equiv\left\langle p_{i}^{-} \mid p_{j}^{+}\right\rangle,[i j] \equiv$ $\left\langle p_{i}^{+} \mid p_{j}^{-}\right\rangle$, where $\left|p_{i}^{ \pm}\right\rangle$is the Weyl spinor for a massless particle with momentum $p_{i}$.

This implies

$$
\begin{array}{r}
\mathcal{M}^{++}=\frac{[21]}{\langle 12\rangle}\left(A-\frac{\operatorname{tr}^{-}(1323)}{2 s_{12}} B_{33}-\frac{\operatorname{tr}^{-}(1423)}{2 s_{12}} B_{34}\right. \\
\left.-\frac{\operatorname{tr}^{-}(1324)}{2 s_{12}} B_{43}-\frac{\operatorname{tr}^{-}(1424)}{2 s_{12}} B_{44}\right)
\end{array}
$$

and

$$
\begin{aligned}
\mathcal{M}^{+-} & =\frac{\left\langle 2^{-}|3| 1^{-}\right\rangle}{\left\langle 1^{-}|3| 2^{-}\right\rangle}\left(\frac{\operatorname{tr}^{-}(1323)}{2 s_{12}} B_{33}+\frac{\operatorname{tr}^{-}(1324)}{2 s_{12}} B_{34}\right) \\
& +\frac{\left\langle 2^{-}|4| 1^{-}\right\rangle}{\left\langle 1^{-}|4| 2^{-}\right\rangle}\left(\frac{\operatorname{tr}^{-}(1423)}{2 s_{12}} B_{43}+\frac{\operatorname{tr}^{-}(1424)}{2 s_{12}} B_{44}\right) .
\end{aligned}
$$

Contrary to the ++ case, it is not possible to factor out a global spinorial phase in the +- case without introducing denominators that in general aggravate numerical problems.

\subsection{Evaluation of the amplitude coefficients}

Our goal was the analytical reduction of all diagrams, to allow for algebraic cancellations of numerically dangerous denominators in the amplitude. These denominators are so-called Gram determinants which are induced by reduction algorithms of Lorentz tensor integrals. After generating all diagrams, using the QGRAF [47] program, we used FORM 3.1 [48] to perform the gamma matrix algebra and to project the diagrams on the helicity components and amplitude coefficients. Further, by applying the reduction algorithms for scalar and tensor integrals described in $[42,43]$, we expressed all amplitude coefficients as a linear combination of scalar integrals. As scalar integral basis we chose 2-, 3- and 4-point functions $\left(s_{i j}=\left(p_{i}+p_{j}\right)^{2}\right)$ :

$$
\begin{aligned}
& I_{2}^{d=n}\left(s_{i j}, m_{q}^{2}, m_{q}^{2}\right), \\
& I_{3}^{d=4}\left(s_{i j}, s_{k l}, s_{p r}, m_{q}^{2}, m_{q}^{2}, m_{q}^{2}\right), \\
& I_{4}^{d=6}\left(s_{i j}, s_{k l}, s_{p r}, m_{q}^{2}, m_{q}^{2}, m_{q}^{2}, m_{q}^{2}\right),
\end{aligned}
$$

which were evaluated using LoopTools-2.2 [49]. The spurious UV pole of the 2-point integral cancels when adding all diagrams. The full amplitude is composed out of 12, 24 and 31 different 2-, 3-, 
and 4-point functions. The complexity of the expressions is induced by the number of independent scales, which is seven here. One may chose $s_{12}, s_{23}, s_{34}, s_{45}, s_{15}, m_{q}^{2}, m_{h}^{2}$. The coefficient of each function was exported to MAPLE to apply simplification algorithms. Schematically,

$$
\mathcal{M}^{\lambda_{1} \lambda_{2}}=\sum_{k} \operatorname{simplify}\left[C_{k}^{\lambda_{1} \lambda_{2}}\right] I_{k} \quad, \quad I_{k} \in\left\{I_{2}^{n}, I_{3}^{4}, I_{4}^{6}\right\} .
$$

In this way, we could achieve expressions with a simple denominator structure allowing for a stable numerical evaluation. In the equal helicity case, $\lambda_{1}=\lambda_{2}$, all Gram determinants cancel. In the opposite helicity case, $\lambda_{1}=-\lambda_{2}$, one Gram determinant survives. The simplified expressions were then exported to Fortran code. Each of these steps was completely automatized.

\subsection{Numerical implementation}

In order to compute numerical results for hadron colliders, the differential partonic cross section has to be convoluted with parton distribution functions (PDFs) and integrated over the $2 \rightarrow 3$-particle phase space. We employed the gluon density of the MRST2002nlo PDF set [50], as implemented in LHAPDF [51], which also provides the strong coupling constant as function of the renormalisation scale. In the MSSM case, where the heavy CP-even Higgs boson $H$ can be resonant, we used multichannel Monte Carlo (MC) integration techniques $[52,53]$ with phase space mappings based on $[54,55]$ and the adaptive MC integration package BASES [56]. The relevant quartic Higgs couplings were implemented in the program HDECAY [37], which incorporates the routine FeynHiggsFast [57], in order to evaluate the radiatively corrected Yukawa- and Higgs couplings and also the Higgs widths, as discussed above.

\section{Results}

In this section we present and discuss the LHC cross sections for 2- and 3-Higgs boson production. We use the following parameters throughout:

$$
\begin{aligned}
\alpha_{s}\left(M_{Z}\right) & =0.120 \\
\alpha(0) & =1 / 137.036 \\
m_{t} & =178 \mathrm{GeV} \\
m_{b} & =4.7 \mathrm{GeV} \\
m_{W} & =80.41 \mathrm{GeV} \\
m_{Z} & =91.1875 \mathrm{GeV}
\end{aligned}
$$

For 2-Higgs (3-Higgs) boson production, the factorisation scale $\mu_{F}$ and renormalisation scale $\mu_{R}$ were set to $\mu_{F}=\mu_{R}=2 m_{H}\left(3 m_{H}\right)$. The strong coupling constant $\alpha_{s}$ was taken at $\mu_{R}$, but for the fine structure constant we used $\alpha(0)$. All results have been calculated with a MC error of $0.5 \%$ or less.

In the following subsections we present SM cross sections for 2- and 3-Higgs boson production and describe how BSM scenarios allow for observable enhancements of the SM rates.

\subsection{Multi-Higgs boson production in the SM}

We begin with the gluon-fusion production cross section for 2 and 3 Higgs bosons. Note that in both cases large next-to-leading order corrections are expected, leading to $K$-factors as large as 2 like in the case of single Higgs boson production [58,59], since the infrared structure of these processes is identical (with a large contribution from soft gluon effects). 


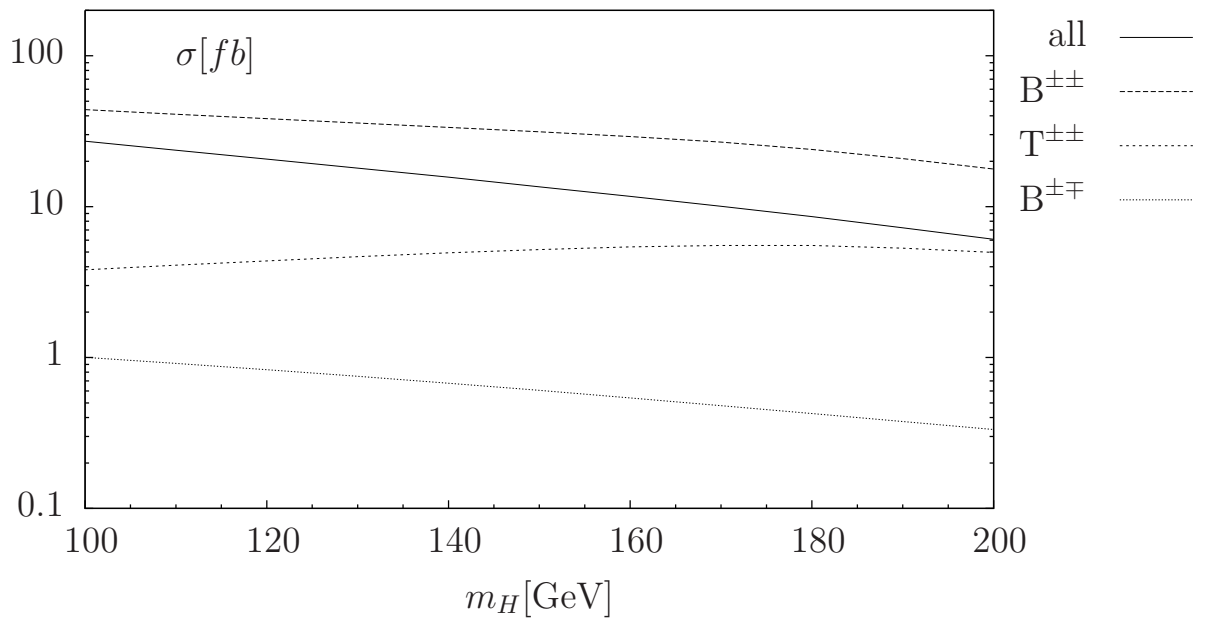

Figure 2: Total Higgs pair production cross section vs. $m_{H}$ at the LHC, as well as the individual cross sections for box (B) and triangle (T) topologies with equal $(++/--)$ and opposite $(+-/-+)$ helicity components.

In Fig. 2. we display the Higgs pair production cross section vs. $m_{H}$ at $\sqrt{s}=14 \mathrm{TeV}$. Our results show good agreement with [23] when PDF, scale and parameter uncertainties are taken into account. The total cross section falls from about 30 to $6 \mathrm{fb}$ in the Higgs mass range from 100 to $200 \mathrm{GeV}$. In addition to the total cross section, the equal $++/--$ and opposite $+-/-+$ helicity components of the cross section are also shown. The triangle topologies only allow for a $L=S=0$ interaction, i.e. the $+-/-+$ helicity component is zero. Overall, the opposite helicity component is more than an order of magnitude suppressed. Furthermore, a destructive interference effect is visible between the box and triangle topologies.

In Fig. 3, the total cross section for triple Higgs boson production at the LHC is plotted vs. $m_{H}$. Our results agree with the recent calculation of [26] within MC errors. Again one finds that the opposite helicity components of the cross section are more than an order of magnitude suppressed. In the figure, the pentagon, box, and triangle contributions are also shown separately. The latter, being proportional to $\lambda_{3}^{2}$ and $\lambda_{4}$, are suppressed relative to the box and pentagon topologies. Due to interference effects the contribution of the quartic Higgs self coupling to the total cross section is not negligible: it varies between $+1 \%\left(m_{H}=100 \mathrm{GeV}\right)$ and $-57.5 \%\left(m_{H}=200 \mathrm{GeV}\right)$. The destructive interference pattern between triangle, box and respectively box and pentagon contributions is wellknown. It can be understood from from the fact that the effective two-gluon $n$-Higgs boson operators contain a factor $(-1)^{n}[22]$. As the self couplings increase with increasing Higgs mass, the box and triangle topologies become more and more important relative to the pentagon contribution.

In Table 1 we give predictions for different values of the Higgs mass for the LHC and a $200 \mathrm{TeV}$ Very Large Hadron Collider (VLHC). We also note that a change in the top mass best fit value from $m_{t}=178 \mathrm{GeV}$ to $172.5 \mathrm{GeV}$ leads to a $15 \%$ decrease of the cross section.

In Table 2. we compare the relative importance of the different topologies and helicities at the VLHC.

Furthermore, the gluon fusion cross section is proportional to $\alpha_{s}^{2}$ and thus very sensitive to renormalisation scale variations, as exemplified in Fig. 4 for $\mu=\mu_{R}=\mu_{F}$. Here, the scale $\mu$ is varied around the central choice $\mu_{0}=2 m_{H}$ and $\mu_{0}=3 m_{H}$ for 2- and 3-Higgs boson production, respectively, by a factor $\mu / \mu_{0} \in[1 / 3,3]$. From this we estimate a scale uncertainty of about $50 \%$. Thus, large $K$-factors due to higher order effects can be expected. In Table 3 we study the scale 


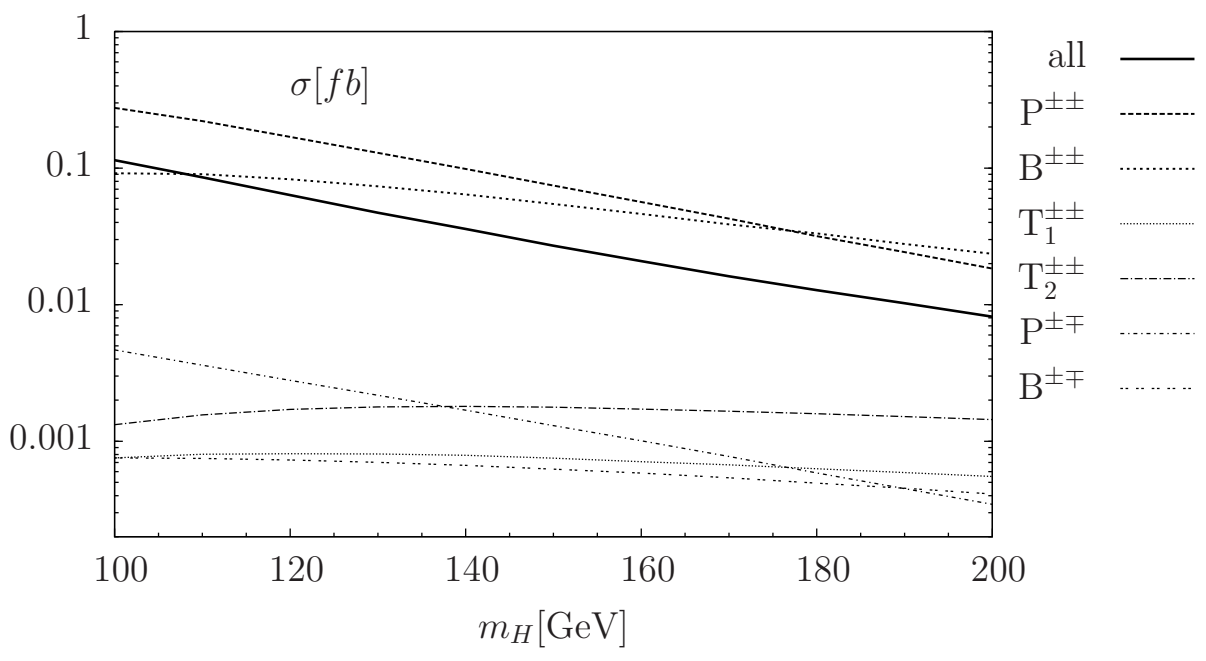

Figure 3: The total 3-Higgs boson production cross section vs. $m_{H}$ at the LHC. Equal and opposite helicity components of contributions from various topologies are shown.

\begin{tabular}{ccccc}
\hline \hline & $m_{H}[\mathrm{GeV}]$ & 120 & 150 & 180 \\
\hline LHC & $\sigma[\mathrm{fb}]$ & 0.0623 & 0.0267 & 0.0126 \\
VLHC & $\sigma[\mathrm{fb}]$ & 9.55 & 4.89 & 2.98 \\
\hline \hline
\end{tabular}

Table 1: Typical cross sections for triple Higgs boson production at the LHC and a $200 \mathrm{TeV}$ VLHC.

dependence of the cross section for 3-Higgs boson production by varying the renormalisation and factorisation scales independently for $m_{H}=120 \mathrm{GeV}$. Note that the gluon luminosity decreases with increasing scale $\mu_{F}$, because in 3-Higgs boson production the momentum of the gluons has to be relatively high. Hence, the cross section shrinks with increasing $\mu_{F}$ and $\mu_{R}$. The table demonstrates that varying $\mu_{R}$ and $\mu_{F}$ in the same direction yields a conservative estimate of the scale uncertainty.

Many years ago multi-Higgs boson production via gluon-fusion was studied in [60] in the heavy top limit. For single Higgs boson production via gluon fusion this limit is well known to be a good approximation [61]. In the context of multi-Higgs boson production, the heavy top limit has been applied at the leading [60] and next-to-leading level [62]. In [16], the quality of the heavy top approximation has been studied for Higgs pair production, and agreement at the $\mathcal{O}(10 \%)$-level for the total cross section, but large discrepancies for kinematic distributions have been observed when comparing results for $m_{t} \rightarrow \infty$ and physical $m_{t}$. In Fig. [5 we compare our physical- $m_{t}$ results with results in the heavy top limit. While there is reasonable agreement in the 2-Higgs case for small

\begin{tabular}{cccccccc}
\hline \hline$\sigma[\mathrm{fb}]$ & all & $P^{ \pm \pm}$ & $B^{ \pm \pm}$ & $T_{1}^{ \pm \pm}$ & $T_{2}^{ \pm \pm}$ & $P^{ \pm \mp}$ & $B^{ \pm \mp}$ \\
\hline$m_{H}=120 \mathrm{GeV}$ & 9.55 & 21.82 & 10.04 & 0.111 & 0.189 & 0.589 & 0.169 \\
$m_{H}=200 \mathrm{GeV}$ & 1.93 & 3.97 & 4.76 & 0.129 & 0.262 & 0.125 & 0.163 \\
\hline \hline
\end{tabular}

Table 2: Contributions of different topologies to triple Higgs boson production at a $200 \mathrm{TeV}$ VLHC. 

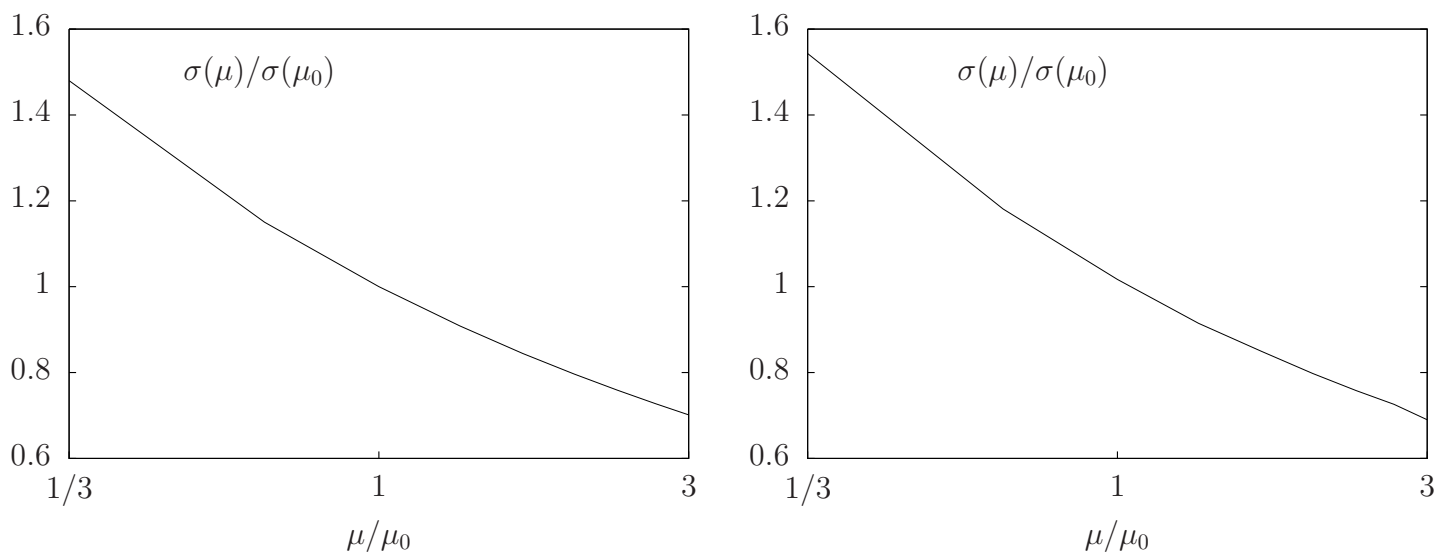

Figure 4: Renormalisation and factorisation scale dependence $\left(\mu=\mu_{R}=\mu_{F}\right)$ of the 2-Higgs (left, $\mu_{0}=2 m_{H}$ ) and 3-Higgs (right, $\mu_{0}=3 m_{H}$ ) cross sections at the LHC.

\begin{tabular}{cccc}
\hline \hline$\sigma\left[10^{-2} \mathrm{fb}\right]$ & $\mu_{F}=m_{H}$ & $3 m_{H}$ & $9 m_{H}$ \\
\hline$\mu_{R}=m_{H}$ & 9.71 & 8.61 & 7.66 \\
$3 m_{H}$ & 7.21 & 6.38 & 5.68 \\
$9 m_{H}$ & 5.57 & 4.93 & 4.39 \\
\hline \hline
\end{tabular}

Table 3: Renormalisation and factorisation scale dependence of the $g g \rightarrow H H H$ cross section for $m_{H}=120 \mathrm{GeV}$ at the LHC.

Higgs masses, the heavy top limit fails completely in the 3-Higgs case. To better understand this observation, we study the variation of the cross sections with the internal quark mass $m_{q}$ for a fixed value of $m_{H}=120 \mathrm{GeV}$ in Fig. 6. We see that in the 2-Higgs case the heavy top limit accidentally agrees with the result for $m_{q}=m_{t}$ (indicated by the vertical line). However, asymptotically the result for finite $m_{q}$ approaches the $m_{q} \rightarrow \infty$ limit only for masses around $3 \mathrm{TeV}$. The same holds for the 3-Higgs case, but here the result for $m_{q}=m_{t}$ is an order of magnitude smaller than the heavy top limit. In both cases the dominant contribution to the cross section comes from the kinematic regime close to the top pair threshold $s_{i j} \sim 4 m_{t}^{2}$. We conclude that the heavy top limit is not applicable when calculating multi-Higgs boson production cross sections and should not be used in experimental studies.

\subsection{Multi-Higgs boson production beyond the SM}

If one allows for higher dimensional operators in the Higgs sector the trilinear and quartic Higgs self couplings are no longer directly related to the Higgs mass. This has motivated us to study the variation of the cross section with respect to $\lambda_{3}, \lambda_{4}$ and $m_{H}$. In Fig. 7 we illustrate the variation of the cross section for 3-Higgs boson production with $\lambda_{3}$ and $\lambda_{4}\left(m_{H}\right.$ is fixed), and in Fig. 8 the variation with $\lambda_{3}$ and $m_{H}\left(\lambda_{4}\right.$ is fixed).

The variation of the cross section with $\lambda_{3}, \lambda_{4}$ and $m_{H}$ is mainly due to phase space and interference effects. When $m_{H}$ increases, the phase space is reduced and the PDF and $\alpha_{s}$ are to be taken at a higher value of $x$ and a larger scale. All effects conspire and lead to a smaller cross section. The dependence on $\lambda_{3}$ is mainly due to the interference pattern. In Fig. 9 a slice of the contour plot Fig. 8 is shown for $m_{H}=160 \mathrm{GeV}$. When the trilinear coupling $\lambda_{3}$ becomes negative, the destructive interference between box and pentagon topologies turns into a constructive one, which explains the 

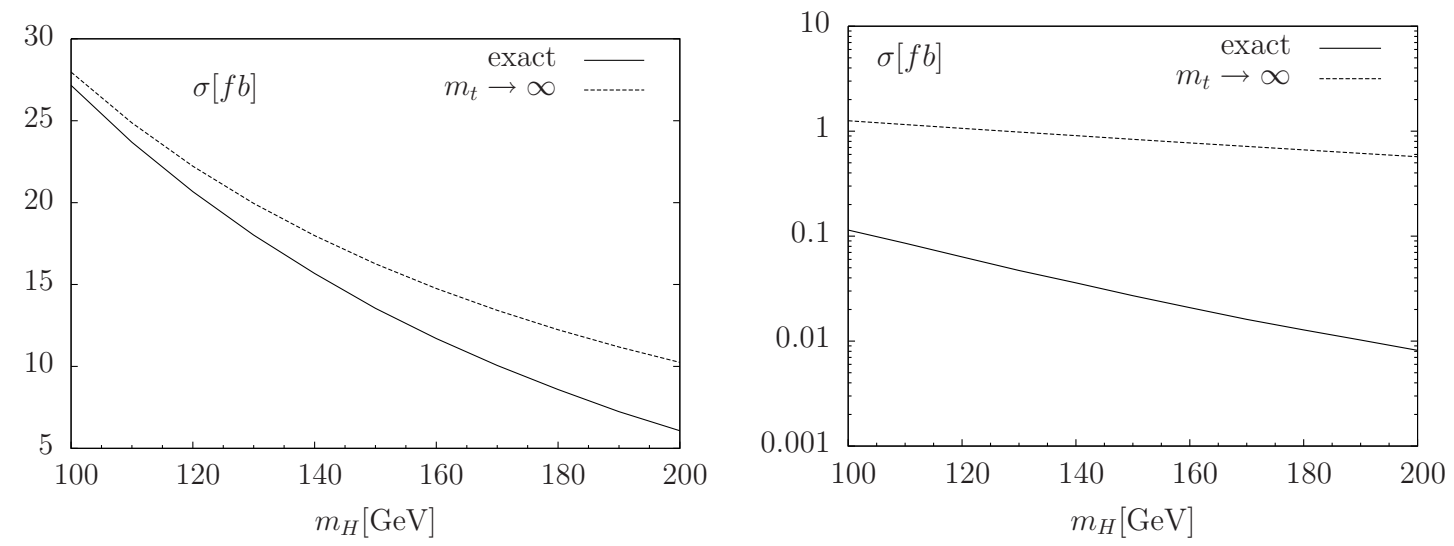

Figure 5: Comparison of the total cross section for 2-Higgs (left) and 3-Higgs (right) boson production vs. $m_{H}$ at the LHC, calculated for the physical value of $m_{t}$ and in the $m_{t} \rightarrow \infty$ limit.
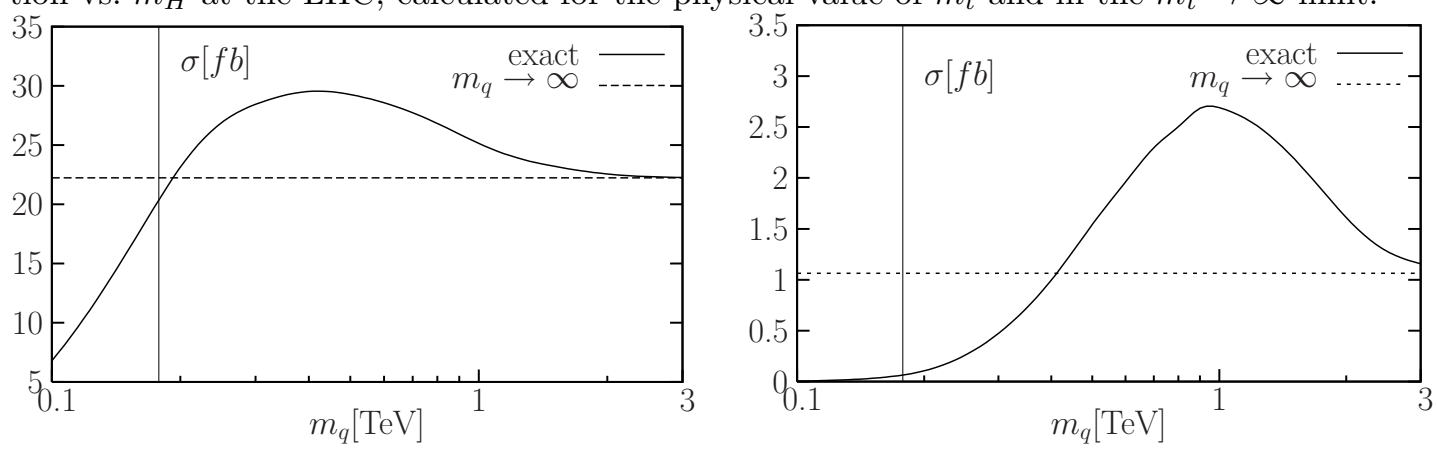

Figure 6: The total cross section for 2-Higgs (left) and 3-Higgs (right) production vs. $m_{q}$ at the LHC. The $m_{q} \rightarrow \infty$ limit is shown as horizontal line. The vertical line indicates the actual value of the top quark mass.

rise of the cross section in this regime. Increasing the coupling to positive values beyond the SM value first enhances the destructive interference effect, but finally the box topologies win and the full cross section grows again. The minimum moves to lower values of $\lambda_{3}$ when the Higgs mass gets larger, due to the growth of the Higgs self-couplings. We note that the same effect happens in the 2-Higgs case. It implies that even a precise measurement of the cross section alone would not lead to a unique determination of the trilinear coupling.

This reasoning allows now to understand the variation of the cross section with $\lambda_{4}$ in Fig. 7 . For negative values of $\lambda_{3}$ the box and pentagon topologies interfere constructively and the relative importance of the triangle contribution proportional to $\lambda_{4}, \lambda_{3}^{2}$ is further reduced, resulting in an almost flat dependence on $\lambda_{4}$. For positive $\lambda_{3}$ the contribution of the triangle topologies is pronounced by destructive interferences between the various topologies leading to a slight variation with $\lambda_{4}$. As can be seen from Fig. [ for $\lambda_{3} / \lambda_{3}^{\mathrm{SM}}$ in the range 0.5 to 1.5 and $\lambda_{4} / \lambda_{4}^{\mathrm{SM}}$ in the range -3 to 3 the cross section varies from 0.03 to $0.1 \mathrm{fb}$.

In principle the couplings are restricted only by unitarity bounds. To illustrate the effects of large couplings that approach the non-perturbative regime, we list in Table 4 cross sections for values $\left|\lambda_{3} / v\right|,\left|\lambda_{4}\right| \sim 4 \pi$. We see that non-perturbative effects in the Higgs sector may well lead to sizable triple Higgs cross sections of up to $30 \mathrm{fb}$ at the LHC. 


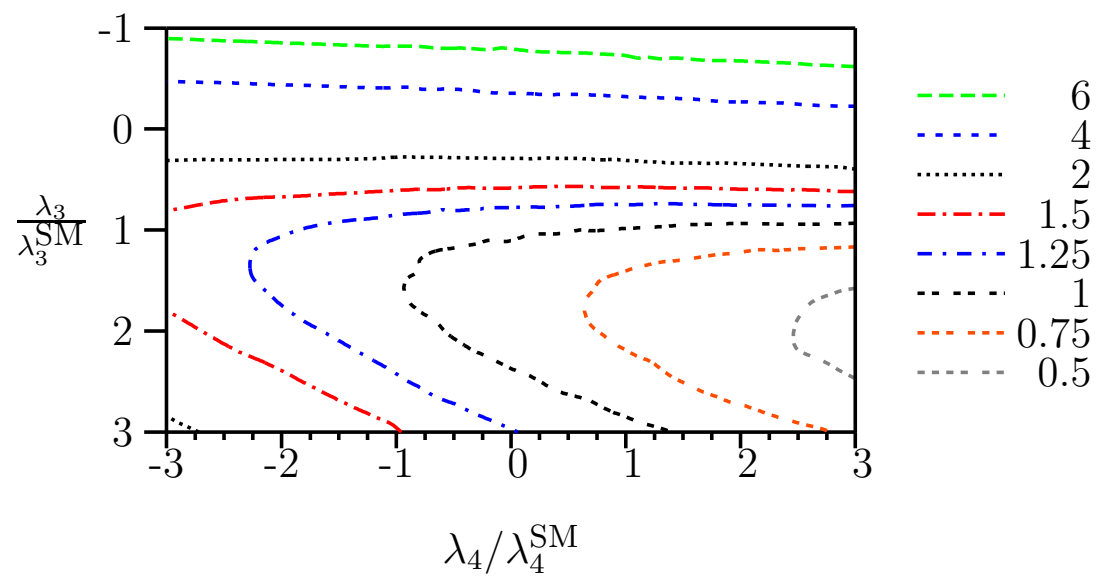

Figure 7: Contour plot showing the variation of the cross section for 3-Higgs boson production with $\lambda_{3}$ and $\lambda_{4}$ for $m_{H}=120 \mathrm{GeV}$ at the LHC. The numbers denote the cross section normalised to $\sigma_{\mathrm{SM}}\left(m_{H}=120 \mathrm{GeV}\right)=0.0623 \mathrm{fb}$.

As already pointed out in Section 2, in 2HDMs one finds two amplification effects for multi-Higgs boson production. Both effects are illustrated in Fig. 10 for the MSSM. For small $\tan \beta=3$ the heavy CP even Higgs boson may become resonant in some of the topologies sketched in Fig. 1). This is illustrated in Fig. 10 a. With the given choice of parameters one sees that for $m_{h}>109 \mathrm{GeV}$ the $H \rightarrow h$ channel opens up inducing a resonant amplification of the box and triangle topologies, $B$ and $T_{2}$. For $m_{h}>111 \mathrm{GeV}$ also the $H \rightarrow h h h$ channel opens up leading to an enhancement of the triangle topology $T_{1}$ proportional to the quartic coupling $\lambda_{H h h h}$. Since this triangle contribution is suppressed relative to the other topologies the effect is hardly visible in the plot (tiny peak next to the right vertical line). We see that in BSM scenarios resonant amplification may lead to triple Higgs production reaching a few fb (not taking into account the expected $K$-factor of 2 ), which would be observable at the SuperLHC or even at the LHC.

For large $\tan \beta=50$ the top contribution is largely suppressed and the bottom loops become dominant due to the enhanced Yukawa couplings. Resonance effects on the other hand are now

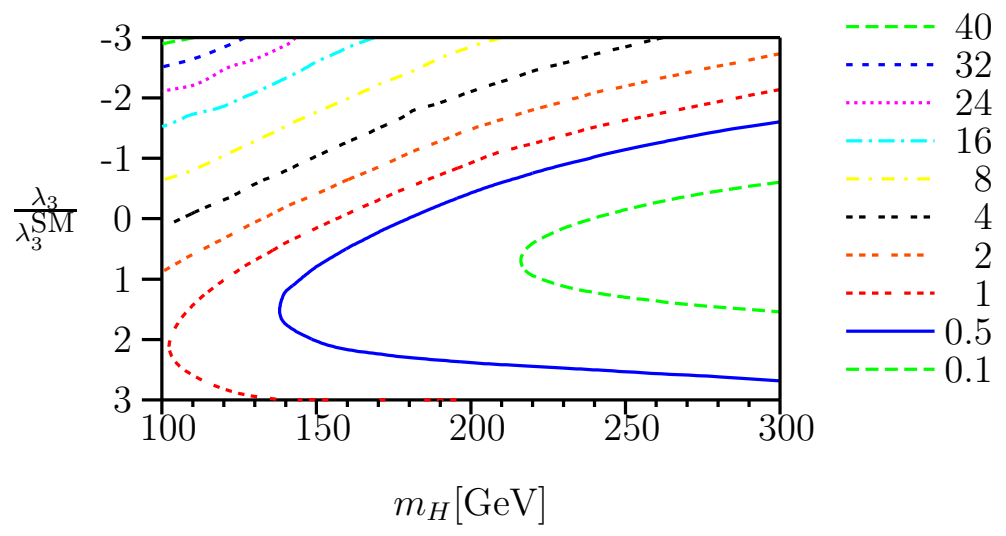

Figure 8: Contour plot showing the variation of the cross section for 3-Higgs boson production with $\lambda_{3}$ and $m_{H}$ for $\lambda_{4}=\lambda_{4}^{S M}$ at the LHC, normalised to $\sigma_{\mathrm{SM}}\left(m_{H}=120 \mathrm{GeV}\right)=0.0623 \mathrm{fb}$. 


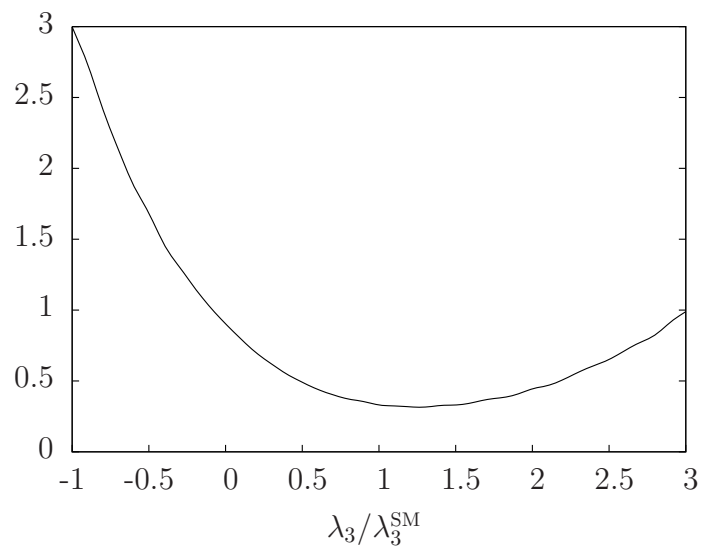

Figure 9: Variation of the cross section for 3-Higgs boson production with $\lambda_{3}$ for $m_{H}=160 \mathrm{GeV}$ and fixed $\lambda_{4}=\lambda_{4}^{\mathrm{SM}}$ at the LHC, normalised to $\sigma_{\mathrm{SM}}\left(m_{H}=120 \mathrm{GeV}\right)=0.0623 \mathrm{fb}$.

\begin{tabular}{cccc}
\hline \hline$\sigma[\mathrm{fb}]$ & $\lambda_{4}=-4 \pi$ & 0 & $4 \pi$ \\
\hline$\lambda_{3} / v=-4 \pi$ & 28.0 & 30.7 & 33.4 \\
0 & 0.169 & 0.0271 & 0.0428 \\
$4 \pi$ & 12.2 & 14.0 & 15.8 \\
\hline \hline
\end{tabular}

Table 4: 3-Higgs boson production cross sections for extreme choices of the Higgs couplings for $m_{H}=150 \mathrm{GeV}$ at the LHC.

negligible, because the Higgs width $\Gamma_{H}$ is a factor 50 larger than at small $\tan \beta=3$. Based on the double Higgs production results in [21], we expect therefore that the LO triple Higgs production cross section in bottom quark fusion is smaller than in gluon fusion. As the pentagon contribution to the cross section is proportional to $\lambda_{h b \bar{b}}^{6}$, all other topologies are much less relevant. For $m_{A} \rightarrow \infty$, $m_{h}$ approaches its maximal value. In this limit one obtains the SM value for the cross section as shown in Fig. 10 $\mathrm{p}$. The minimum around $m_{h} \sim 115 \mathrm{GeV}$ arises due to the interplay of the Yukawaand Higgs self coupling. Although the cross section rises for decreasing $m_{h}$, it is always well below $1 \mathrm{fb}$ in the range considered. Note that in the 2-Higgs boson production case the bottom loops are more pronounced than in the 3-Higgs case, because the scalar loop integrals are probed in different kinematical regions.

In principle, also squark loops have to be considered to obtain a complete prediction for $h h h-$ production in the MSSM. In the heavy squark limit this contribution decouples and approaches zero in contrast to the quark contribution. For the present illustration of amplification effects we have neglected squark effects. 

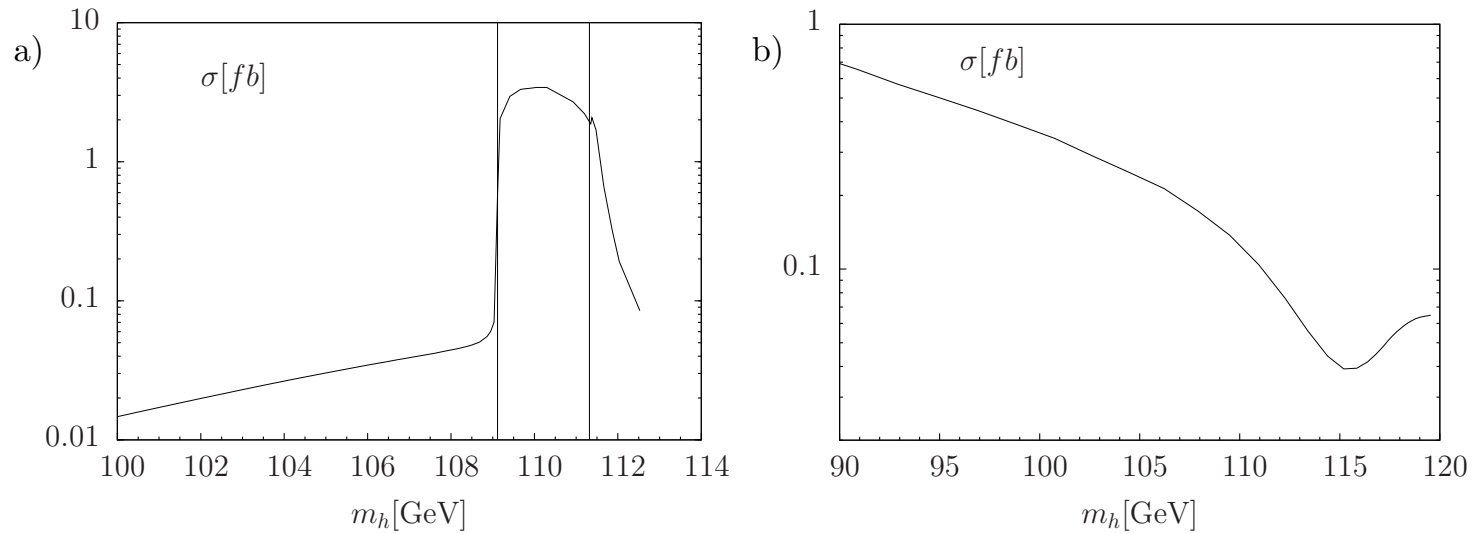

Figure 10: Cross section for 3-Higgs boson production vs. $m_{h}$ at the LHC in the MSSM for $\tan \beta=3$ (left) and $\tan \beta=50$ (right) including mixing effects $\left(A_{t}=1 \mathrm{TeV}, \mu / \mathrm{TeV}=-1(+1)\right.$ for $\tan \beta=$ $3(50))$. The vertical lines in the left plot indicate the 2 - and 3 -Higgs boson thresholds $(H \rightarrow h h$ and $H \rightarrow h h h)$. 


\section{Summary}

We presented our calculation of the loop-induced processes $g g \rightarrow H H$ and $g g \rightarrow H H H$, and discussed the resulting cross sections and their experimental accessibility at the LHC in the SM and beyond. The contributions from pentagon, box and triangle topologies exhibit strong interference patterns and large differences in the equal and opposite gluon helicity components. Generally, the opposite helicity component, which corresponds to a gluon pair with helicity 2, is suppressed by more than an order of magnitude. Furthermore, the triangle topologies are suppressed relative to the box and pentagon topologies. This results in a complex dependence of the cross sections on the trilinear and quartic Higgs self couplings. The cross section for 3-Higgs boson production varies strongly with the quartic Higgs self coupling. For $m_{H}=100 \mathrm{GeV}$ and $200 \mathrm{GeV}$ it affects the cross section by $+1 \%$ and $-57.5 \%$, respectively. This effect is due to the fact that the Higgs self coupling contributions are enhanced for higher Higgs masses and that there is a strong destructive interference between box and pentagon contributions. The estimated renormalisation and factorisation scale uncertainty of about $50 \%$ (variation by a factor 3) is typical for leading-order QCD cross sections. We also investigated the applicability of the heavy top quark approximation for 2- and 3-Higgs boson production and demonstrated that the heavy top limit is not applicable when calculating multi-Higgs boson production cross sections. In summary, we find that the SM cross sections for 3-Higgs boson production are too small for observation at the LHC. The measurement of the SM trilinear Higgs self coupling in 2-Higgs boson production, on the other hand, may be feasible at a luminosity upgraded LHC, termed SuperLHC, collecting $6000 \mathrm{fb}^{-1}$ of data, as long as QCD backgrounds are not prohibitively large $[8,63]$.

The experimental prospects improve if favourable extensions of the SM are realized in nature. We have demonstrated that cross sections for triple Higgs boson production can be as large as $\mathcal{O}(10) \mathrm{fb}$ if one allows for higher dimensional operators or considers 2-Higgs-doublet models, e.g. the MSSM. Here, two amplification effects have been analysed. First, we studied Yukawa coupling enhancements through mixing that lead to important bottom loop contributions. For the 3-Higgs case the cross section remains below $1 \mathrm{fb}$ for the parameters considered and $\tan \beta=50$. Secondly, we demonstrated that resonance effects due to an internal heavy Higgs boson decaying into 2 or 3 light Higgs bosons can enhance the 3-Higgs cross section into the potentially observable $\mathcal{O}(\mathrm{fb})$ region. Higher dimensional operators lead to essentially unconstrained trilinear and quartic couplings. Strong coupling of the order $\left|\lambda_{3} / v\right|,\left|\lambda_{4}\right| \sim 4 \pi$ leads to cross sections of up to $30 \mathrm{fb}$. This implies that already the LHC should be able to restrict the $\lambda_{3}-\lambda_{4}$ plane. Although these bounds will not be very restrictive, they may still exclude parameter regions that exhibit non-perturbative effects, which would be an important qualitative finding. A more stringent bound on $\lambda_{3}$ alone will be obtained from Higgs pair production. We conclude that multi-Higgs boson production at the luminosity-upgraded LHC is an interesting probe of Higgs sectors beyond the SM and warrants further study. A $200 \mathrm{TeV}$ VLHC would of course further improve the sensitivity.

\section{Acknowledgements}

S.K. thanks the PPT group at the University of Edinburgh for their hospitality while this paper was completed. T.B. thanks F. Boudjema for discussion. This work was supported by the Deutsche Forschungsgemeinschaft (DFG) under contract number BI 1050/1, the DFG Research Training Group 1147, the Scottish Universities Physics Alliance (SUPA), and the Bundesministerium für Bildung und Forschung (BMBF, Bonn, Germany) under contract number 05HT1WWA2. 


\section{References}

[1] P. W. Higgs, Phys. Lett. 12 (1964) 132; P. W. Higgs, Phys. Rev. 145 (1966) 1156; F. Englert and R. Brout, Phys. Rev. Lett. 13 (1964) 321; G. S. Guralnik, C. R. Hagen and T. W. B. Kibble, Phys. Rev. Lett. 13 (1964) 585.

[2] V. Buscher and K. Jakobs, Int. J. Mod. Phys. A 20 (2005) 2523 arXiv:hep-ph/0504099.

[3] A. Djouadi, arXiv:hep-ph/0503172 and arXiv:hep-ph/0503173.

[4] T. Binoth and A. Ghinculov, Nucl. Phys. B 550 (1999) 77 arXiv:hep-ph/9808393.

[5] A. Ghinculov, T. Binoth and J. J. van der Bij, Phys. Lett. B 427 (1998) 343 arXiv:hep-ph/9802367.

[6] R. Barate et al. [LEP Working Group for Higgs boson searches], Phys. Lett. B 565 (2003) 61 arXiv:hep-ex/0306033.

[7] M. Dührssen, S. Heinemeyer, H. Logan, D. Rainwater, G. Weiglein and D. Zeppenfeld, Phys. Rev. D 70 (2004) 113009 arXiv:hep-ph/0406323.

[8] G. Weiglein et al. [LHC/LC Study Group], "Physics interplay of the LHC and the ILC," arXiv:hep-ph/0410364

[9] A. Djouadi, W. Kilian, M. Muhlleitner and P. M. Zerwas, Eur. Phys. J. C 10 (1999) 27 arXiv:hep-ph/9903229].

[10] M. Battaglia, E. Boos and W. M. Yao, in Proc. of the APS/DPF/DPB Summer Study on the Future of Particle Physics (Snowmass 2001) ed. N. Graf, eConf C010630 (2001) E3016 arXiv:hep-ph/0111276.

[11] A. Gutierrez-Rodriguez, M. A. Hernandez-Ruiz and O. A. Sampayo, arXiv:hep-ph/0601238.

[12] G. V. Jikia, Nucl. Phys. B 412 (1994) 57.

[13] F. Boudjema and E. Chopin, Z. Phys. C 73 (1996) 85 arXiv:hep-ph/9507396.

[14] R. Belusevic and G. Jikia, Phys. Rev. D 70 (2004) 073017 arXiv:hep-ph/0403303.

[15] A. Djouadi, W. Kilian, M. Muhlleitner and P. M. Zerwas, Eur. Phys. J. C 10 (1999) 45 arXiv:hep-ph/9904287.

[16] U. Baur, T. Plehn and D. L. Rainwater, Phys. Rev. D 67 (2003) 033003 arXiv:hep-ph/0211224.

[17] U. Baur, T. Plehn and D. L. Rainwater, Phys. Rev. Lett. 89 (2002) 151801 arXiv:hep-ph/0206024.

[18] U. Baur, T. Plehn and D. L. Rainwater, Phys. Rev. D 68 (2003) 033001 arXiv:hep-ph/0304015.

[19] U. Baur, T. Plehn and D. L. Rainwater, Phys. Rev. D 69 (2004) 053004 arXiv:hep-ph/0310056.

[20] S. Dawson, C. Kao, Y. Wang and P. Williams, arXiv:hep-ph/0610284

[21] L. G. Jin, C. S. Li, Q. Li, J. J. Liu and R. J. Oakes, Phys. Rev. D 71 (2005) 095004 arXiv:hep-ph/0501279.

[22] E. W. N. Glover and J. J. van der Bij, Nucl. Phys. B 309 (1988) 282. 
[23] T. Plehn, M. Spira and P. M. Zerwas, Nucl. Phys. B 479 (1996) 46 [Erratum-ibid. B 531 (1998) 655] arXiv:hep-ph/9603205].

[24] A. Krause, T. Plehn, M. Spira and P. M. Zerwas, Nucl. Phys. B 519 (1998) 85 arXiv:hep-ph/9707430.

[25] O. Brein and W. Hollik, Eur. Phys. J. C 13 (2000) 175 arXiv:hep-ph/9908529.

[26] T. Plehn and M. Rauch, Phys. Rev. D 72 (2005) 053008 arXiv:hep-ph/0507321].

[27] S. Kanemura, S. Kiyoura, Y. Okada, E. Senaha and C. P. Yuan, Phys. Lett. B 558 (2003) 157 arXiv:hep-ph/0211308.

[28] S. Kanemura, Y. Okada, E. Senaha and C. P. Yuan, Phys. Rev. D 70 (2004) 115002 arXiv:hep-ph/0408364.

[29] T. Appelquist and J. Carazzone, Phys. Rev. D 11 (1975) 2856.

[30] V. Barger, T. Han, P. Langacker, B. McElrath and P. Zerwas, Phys. Rev. D 67 (2003) 115001 arXiv:hep-ph/0301097.

[31] C. O. Dib, R. Rosenfeld and A. Zerwekh, JHEP 0605 (2006) 074 arXiv:hep-ph/0509179.

[32] B. W. Lee, C. Quigg and H. B. Thacker, "Weak Interactions At Very High-Energies: The Role Of The Higgs Boson Mass," Phys. Rev. D 16 (1977) 1519.

[33] T. Binoth and J. J. van der Bij, Z. Phys. C 75 (1997) 17 arXiv:hep-ph/9608245.

[34] J. F. Gunion, H. E. Haber, G. L. Kane and S. Dawson, "The Higgs Hunter's Guide," SCIPP89/13; "Errata for the Higgs hunter's guide," arXiv:hep-ph/9302272

[35] H. E. Haber and R. Hempfling, Phys. Rev. D 48 (1993) 4280 arXiv:hep-ph/9307201.

[36] M. Carena, J. R. Espinosa, M. Quiros and C. E. M. Wagner, "Analytical expressions for radiatively corrected Higgs masses and couplings in the MSSM," Phys. Lett. B 355 (1995) 209 arXiv:hep-ph/9504316.

[37] A. Djouadi, J. Kalinowski and M. Spira, Comput. Phys. Commun. 108 (1998) 56.

[38] F. Boudjema and A. Semenov, Phys. Rev. D 66 (2002) 095007 arXiv:hep-ph/0201219.

[39] T. Binoth, J. P. Guillet and G. Heinrich, Nucl. Phys. B 572 (2000) 361 arXiv:hep-ph/9911342.

[40] T. Binoth, J. P. Guillet and F. Mahmoudi, JHEP 0402 (2004) 057 arXiv:hep-ph/0312334.

[41] T. Binoth, M. Ciccolini, N. Kauer and M. Krämer, JHEP 0503 (2005) 065 arXiv:hep-ph/0503094.

[42] T. Binoth, J. P. Guillet, G. Heinrich, E. Pilon and C. Schubert, JHEP 0510 (2005) 015 arXiv:hep-ph/0504267.

[43] T. Binoth, M. Ciccolini and G. Heinrich, arXiv:hep-ph/0601254.

[44] T. Binoth, A. Guffanti, J. P. Guillet, S. Karg, N. Kauer and T. Reiter, arXiv:hep-ph/0606318.

[45] Z. Xu, D. H. Zhang and L. Chang, Nucl. Phys. B 291 (1987) 392.

[46] L. J. Dixon, arXiv:hep-ph/9507214.

[47] P. Nogueira, J. Comput. Phys. 105 (1993) 279. 
[48] J. A. M. Vermaseren, arXiv:math-ph/0010025.

[49] T. Hahn, Comput. Phys. Commun. 140 (2001) 418 hep-ph/0012260.

[50] A. D. Martin, R. G. Roberts, W. J. Stirling and R. S. Thorne, Eur. Phys. J. C 28 (2003) 455 arXiv:hep-ph/0211080.

[51] http://hepforge.cedar.ac.uk/lhapdf/

[52] F. A. Berends, R. Pittau and R. Kleiss, Nucl. Phys. B 424 (1994) 308 arXiv:hep-ph/9404313.

[53] R. Kleiss and R. Pittau, Comput. Phys. Commun. 83 (1994) 141 arXiv:hep-ph/9405257.

[54] N. Kauer and D. Zeppenfeld, Phys. Rev. D 65 (2002) 014021 arXiv:hep-ph/0107181.

[55] N. Kauer, Phys. Rev. D 67 (2003) 054013 arXiv:hep-ph/0212091.

[56] http://minami-home.kek.jp/

[57] S. Heinemeyer, W. Hollik and G. Weiglein, Comp. Phys. Commun. 124 (2000) 76; M. Frank, S. Heinemeyer, W. Hollik and G. Weiglein, arXiv:hep-ph/0202166.

[58] S. Dawson, Nucl. Phys. B 359 (1991) 283.

[59] A. Djouadi, M. Spira and P. M. Zerwas, Phys. Lett. B 264 (1991) 440.

[60] E. W. N. Glover and J. J. van der Bij, CERN-TH-5022-88 Presented at 23rd Rencontres de Moriond: Current Issues in Hadron Physics, Les Arcs, France, Mar 13-19, 1988.

[61] M. Spira, A. Djouadi, D. Graudenz and P. M. Zerwas, Nucl. Phys. B 453 (1995) 17 arXiv:hep-ph/9504378.

[62] S. Dawson, S. Dittmaier and M. Spira, Phys. Rev. D 58 (1998) 115012 arXiv:hep-ph/9805244.

[63] F. Gianotti et al., "Physics potential and experimental challenges of the LHC luminosity upgrade," Eur. Phys. J. C 39 (2005) 293 arXiv:hep-ph/0204087. 